\title{
On the local and global well-posedness theory for the KP-I equation
}

\section{Sur le caractère bien posé local et global pour l'équation KP-I}

\author{
Carlos E. Kenig a,b,1 \\ ${ }^{a}$ School of Mathematics, Institute for Advanced Study, Princeton, NJ 08540, USA \\ ${ }^{\mathrm{b}}$ Department of Mathematics, University of Chicago, Chicago, IL 60637, USA
}

Received 28 October 2003; accepted 8 December 2003

Available online 29 July 2004

\begin{abstract}
In this paper we obtain new local and global well-posedness results for the KP-I equation. (C) 2004 L'Association Publications de l'Institut Henri Poincaré. Published by Elsevier B.V. All rights reserved
\end{abstract}

\section{Résumé}

Dans cet article nous obtenons de nouveaux résultats sur le caractère bien posé local et global de l'équation KP-I. (C) 2004 L'Association Publications de l'Institut Henri Poincaré. Published by Elsevier B.V. All rights reserved

Keywords: Local and global well-posedness; KP-I equation

\section{Introduction}

In this note we prove a new local well-posedness theorem for the KP-I equation (1.2), and use the well-known conservation laws to establish a new global well-posedness result as a consequence. These, our main results, are Theorems 3.1 and 3.4.

As has been shown in [13-15], the KP-I equation is badly behaved with respect to Picard iterative methods in the standard Sobolev spaces and the natural energy space, since the flow map fails to be $C^{2}$ at the origin in these spaces. Thus, one is left with two choices: one either considers function spaces that are different from the standard Sobolev spaces, and then shows that the Picard iteration scheme does apply for them (this is the strategy

\footnotetext{
E-mail address: cek@math.uchicago.edu (C.E. Kenig).

${ }^{1}$ Supported in part by NSF, and at IAS by The von Neumann Fund, The Weyl Fund, The Oswald Veblen Fund and the Bell Companies Fellowship. 
of [4-6], where weighted spaces are used), or one decides to abandon the Picard iteration scheme and tries a different approach to well-posedness theory, which is the approach taken in the present paper. The KP-I equation arises in water wave theory, as modeling capillary gravity waves, in the presence of strong surface tension effects. Its companion equation, the KP-II equation (where the sign in front of the $\partial_{x}^{-1} \partial_{y}^{2} u$ term in (1.2) is + instead of -) is also of importance in water wave theory, and from the point of view of well-posedness theory it is much better understood, since the above mentioned difficulty with Picard iterative methods does not occur for it. In fact (see [2]) KP-II is known to be both locally and globally well-posed in $L^{2}\left(\mathbb{R}^{2}\right)$, "by Picard iteration", and even in some Sobolev spaces of negative index [18-20]. In [13], the authors use the local well-posedness theorem of [7], obtained by a version of the classical energy method, and they couple it with some of the known (formal) conserved quantities, and an ingenious use of the dispersive estimates of Strichartz type for KP-I (see [17]), to show global well-posedness for KP-I in the space $Z=\left\{\phi \in L^{2}\left(\mathbb{R}^{2}\right):\|\phi\|_{L^{2}}+\left\|\partial_{x}^{3} \phi\right\|_{L^{2}}+\left\|\partial_{y} \phi\right\|_{L^{2}}+\left\|\partial_{x} \partial_{y} \phi\right\|_{L^{2}}+\right.$ $\left.\left\|\partial_{x}^{-1} \partial_{y} \phi\right\|_{L^{2}}+\left\|\partial_{x}^{-2} \partial_{y}^{2} \phi\right\|_{L^{\infty}}<\infty\right\}$. Our contribution comes from improving on the local well-posedness result given by the classical energy estimate, by showing local well-posedness in the space $Y_{S}=\left\{\phi \in L^{2}\left(\mathbb{R}^{2}\right):\|\phi\|_{L^{2}}+\right.$ $\left.\left\|J_{x}^{s} \phi\right\|_{L^{2}}+\left\|\partial_{x}^{-1} \partial_{y} \phi\right\|_{L^{2}}<\infty\right\}$ for $s>3 / 2$, where $\widehat{J}_{x}^{s} f(\xi, \eta)=\left(1+|\xi|^{2}\right)^{s / 2} \hat{f}(\xi, \eta)$. We first observe that, in this context, it is still enough to control $\left\|\partial_{x} u\right\|_{L_{t}^{1} L_{x y}^{\infty}}$ (Lemma 1.3). We then adapt the version of the argument first introduced by Koch and Tzvetkov [12] in the context of the Benjamin-Ono equation, given in [10], also for the Benjamin-Ono equation. This uses the 'interpolation inequality' of Lemma 1.7, which is the main new ingredient of our proof. Another important ingredient of the proof is a 'product theory' Leibniz rule for fractional derivatives, given in Lemma 1.8(ii), which follows from [16]. Once our local well-posedness result is established, we use the results in [13], to establish global well-posedness in

$$
Z_{0}=\left\{\phi \in L^{2}\left(\mathbb{R}^{2}\right):\|\phi\|_{L^{2}}+\left\|\partial_{x}^{-1} \partial_{y} \phi\right\|_{L^{2}}+\left\|\partial_{x}^{2} \phi\right\|_{L^{2}}+\left\|\partial_{x}^{-2} \partial_{y}^{2} \phi\right\|_{L^{2}}<\infty\right\} .
$$

The question of the local and global well-posedness of KP-I in the energy space $Y=\left\{\phi \in L^{2}\left(\mathbb{R}^{2}\right):\|\phi\|_{L^{2}}+\right.$ $\left.\left\|\partial_{x} \phi\right\|_{L^{2}}+\left\|\partial_{x}^{-1} \partial_{y} \phi\right\|_{L 2}<\infty\right\}$ remains a challenging open problem.

\section{Preliminary estimates}

We introduce the space, for $s \in \mathbb{R}$,

$$
H_{-1}^{s}\left(\mathbb{R}^{2}\right)=\left\{u \in S^{\prime}\left(\mathbb{R}^{2}\right):\|u\|_{H_{-1}^{s}\left(\mathbb{R}^{2}\right)}<\infty\right\},
$$

where

$$
\|u\|_{H_{-1}^{s}\left(\mathbb{R}^{2}\right)}=\left(\iint\left(1+|\xi|^{-1}\right)^{2}\left(1+|\xi|^{2}+|\eta|^{2}\right)^{s} \cdot|\hat{u}(\xi, \eta)|^{2} d \xi d \eta\right)^{1 / 2}
$$

and $H_{-1}^{\infty}\left(\mathbb{R}^{2}\right)=\bigcap_{s \geqslant 0} H_{-1}^{s}\left(\mathbb{R}^{2}\right)$. We recall a local well-posedness result of Iorio and Nunes [7]. For $u \in H_{-1}^{s}\left(\mathbb{R}^{2}\right)$, $s \geqslant 2$ we define $\partial_{x}^{-1} \widehat{\partial_{y}^{2} u}(\xi, \eta)=-\eta^{2} / \xi \hat{u}(\xi, \eta)$.

Lemma 1.1. The Cauchy problem

$$
\left\{\begin{array}{l}
\partial_{t} u+\partial_{x}^{3} u-\partial_{x}^{-1} \partial_{y}^{2} u+u \partial_{x} u=0 \\
\left.u\right|_{t=0}=u_{0}
\end{array}\right.
$$

is locally well-posed in $H_{-1}^{s}\left(\mathbb{R}^{2}\right)$, for $s>2$.

Also, recall that, from [14], if, in addition, $\left\|u_{0}\right\|_{Z}<\infty$, where $Z=\left\{\phi \in L^{2}\left(\mathbb{R}^{2}\right):\|\phi\|_{Z}<\infty\right\}$, and

$$
\|\phi\|_{Z}=\left\|\phi_{L^{2}}+\right\| \partial_{x}^{3} \phi\left\|_{L^{2}}+\right\| \partial_{y} \phi\left\|_{L^{2}}+\right\| \partial_{x y}^{2} \phi\left\|_{L^{2}}+\right\| \partial_{x}^{-1} \partial_{y} \phi\left\|_{L^{2}}+\right\| \partial_{x}^{-2} \partial_{y}^{2} \phi \|_{L^{2}},
$$

then $u$ in fact extends for all time and remains also in $Z$ for all time. 
Lemma 1.3. Let $u$ be a solution to (1.2), with $u_{0} \in H_{-1}^{\infty}\left(\mathbb{R}^{2}\right) \cap Z$. Let $\widehat{J_{x}^{s f}}(\xi, \eta)=\left(1+|\xi|^{2}\right)^{s / 2} \hat{f}(\xi, \eta)$. Then, for $s \geqslant 0$, we have, for any $T>0$ :

$$
\sup _{0<t<T}\left\|J_{x}^{s} u\right\|_{L_{x y}^{2}}+\left\|\partial_{x}^{-1} \partial_{y} u\right\|_{L_{x y}^{2}} \leqslant C_{s} \exp \left(C_{s} \int_{0}^{T}\left\|\partial_{x} u\right\|_{L_{x y}^{\infty}}\right)\left[\left\|J_{x}^{S} u_{0}\right\|_{L_{x y}^{2}}+\left\|\partial_{x}^{-1} \partial_{y} u_{0}\right\|_{L_{x y}^{2}}\right] .
$$

Proof. We apply $J_{x}^{s}$ to (1.2), multiply by $J_{x}^{s} u$, and integrate in $(x, y)$. We obtain

$$
\partial_{t} \int_{\mathbb{R}^{2}} \frac{\left(J_{x}^{s} u\right)^{2}}{2}-\iint_{\mathbb{R}^{2}} \partial_{x}^{-1} \partial_{y}^{2} J_{x}^{s} u J_{x}^{s} u+\iint_{\mathbb{R}^{2}} \partial_{x}^{3} J_{x}^{s} u J_{x}^{s} u=-\iint J_{x}^{s}\left(u \partial_{x} u\right) J_{x}^{s}(u) .
$$

The last two terms in the left-hand side of the equality are 0 . To bound the right-hand side, we use the Kato and Ponce [9] commutator estimate, in the form

$$
\left\|J^{s}(f g)-f J^{s}(g)\right\|_{L^{2}(\mathbb{R})} \leqslant C_{s} \cdot\left[\left\|\partial_{x} f\right\|_{L^{\infty}(\mathbb{R})}\left\|J^{s-1} g\right\|_{L^{2}(\mathbb{R})}+\left\|J^{s} f\right\|_{L^{2}(\mathbb{R})}\|g\|_{L^{\infty}(\mathbb{R})}\right] .
$$

We thus see that the right-hand side above equals (with $f=u, g=\partial_{x} u$ ), integrating first in $x$, and then in $y$

$$
=-\int u J_{x}^{s} \partial_{x} u . J_{x}^{s} u+\mathrm{O}\left(\left\|\partial_{x} u\right\|_{L^{\infty}\left(\mathbb{R}^{2}\right)}\left\|J_{x}^{s} u\right\|_{L^{2}\left(\mathbb{R}^{2}\right)}^{2}\right) .
$$

Thus,

$$
\frac{d}{d t} \int\left|J_{x}^{s} u\right|^{2} d x d y \leqslant C_{s}\left\|\partial_{x} u\right\|_{L^{\infty}\left(\mathbb{R}^{2}\right)}\left\|J_{x}^{s} u\right\|_{L^{2}\left(\mathbb{R}^{2}\right)}^{2},
$$

and the desired estimate for $\left\|J_{x}^{S} u\right\|_{L^{2}\left(\mathbb{R}^{2}\right)}$ follows. To estimate $\partial_{x}^{-1} \partial_{y} u$, we apply $\partial_{x}^{-1} \partial_{y}$ to (1.2), multiply by $\left(\partial_{x}^{-1} \partial_{y} u\right)$ and integrate. The right-hand side will be, this time,

$$
-\iint \partial_{y}\left(\frac{u^{2}}{2}\right) \cdot \partial_{x}^{-1} \partial_{y} u=-\iint u \partial_{y} u \cdot \partial_{x}^{-1} \partial_{y} u=-\iint u \partial_{x} \partial_{x}^{-1} \partial_{y} u \cdot \partial_{x}^{-1} \partial_{y} u=\iint \frac{\partial_{x} u \partial_{x}^{-1} \partial_{y} u \partial_{x}^{-1} \partial_{y} u}{2},
$$

and the desired estimate follows.

We next recall the Strichartz estimates associated to the free KP-I evolution.

Remark. For future use, for $f \in S^{\prime}\left(\mathbb{R}^{2}\right)$ we define $\widehat{D_{x}^{a} f}(\xi, \eta)=|\xi|^{a} \hat{f}(\xi, \eta), \widehat{D_{y}^{b}} f(\xi, \eta)=|\eta|^{b} \hat{f}(\xi, \eta)$.

Lemma 1.5. Let $U(t)=\exp \left(-t\left(\partial_{x}^{3}-\partial_{x}^{-1} \partial_{y}^{2}\right)\right)$ be the unitary group defining the free KP-I evolution. Then, the following estimates hold

$$
\begin{aligned}
& \|U(t) \phi\|_{L_{T}^{q} L_{x y}^{r}} \lesssim\|\phi\|_{L^{2}}, \\
& \left\|\int_{0}^{t} U\left(t-t^{\prime}\right) F\left(t^{\prime}\right) d t^{\prime}\right\|_{L_{T}^{q} L_{x y}^{r}} \lesssim\|F\|_{L_{T}^{\bar{q}} L_{x y}^{\bar{r}},}
\end{aligned}
$$

where $\frac{1}{q}+\frac{1}{r}=\frac{1}{\bar{q}}+\frac{1}{\bar{r}}-1=\frac{1}{2}, 2 \leqslant r<+\infty, 1<\bar{r} \leqslant 2$.

Note that the constant in the two inequalities of Lemma 1.5 is independent of $T$. The proof of Lemma 1.5 is given in [17]. Combining Lemma 1.5 with a Sobolev-embedding, we obtain 
Corollary 1.6. For each $T>0, \varepsilon>0$, we have

$$
\|U(t) \phi\|_{L_{T}^{2} L_{x y}^{\infty}} \lesssim C_{\varepsilon, T}\left\{\|\phi\|_{L^{2}}+\left\|D_{x}^{\varepsilon} \phi\right\|_{L^{2}}+\left\|D_{y}^{\varepsilon} \phi\right\|_{L^{2}}\right\}
$$

Our main new linear estimate is contained in the next lemma. It is an adaptation to the present setting of Proposition 2.8 in [10], which in turn is a reformulation of the main estimate in [12]. See also [15] for another use of the [12] idea in the context of KP-I.

Lemma 1.7. Let $\delta>0, T \in(0,1]$. Assume that $w \in C\left([0, T] ; H_{-1}^{3}\left(\mathbb{R}^{2}\right)\right)$ is a solution to the linear equation

$$
\partial_{t} w+\partial_{x}^{3} w-\partial_{x}^{-1} \partial_{y}^{2} w=F
$$

Then,

$$
\begin{aligned}
\left\|\partial_{x} w\right\|_{L_{T}^{1} L_{x y}^{\infty}} \lesssim & C_{\delta, T}\left[\sup _{0<t<T}\left\|J_{x}^{\frac{3}{2}+2 \delta} w\right\|_{L^{2}}+\sup _{0<t<T}\left\|J_{x}^{\frac{3}{2}+\delta} D_{y}^{\delta} w\right\|_{L^{2}}\right. \\
& \left.+\int_{0}^{T}\left\|J_{x}^{1 / 2+2 \delta} F\right\|_{L^{2}}+\left\|J_{x}^{1 / 2+\delta} D_{y}^{\delta} F\right\|_{L^{2}}\right]
\end{aligned}
$$

Proof. We use a Littlewood-Paley decomposition of $w$ in the $\xi$ variable. More precisely, if $\eta \in C_{0}^{\infty}\left(\frac{1}{2}<|\xi|<2\right)$, $\chi \in C_{0}^{\infty}(|\xi|<2)$, are such that $1=\sum_{k=1}^{\infty} \eta\left(2^{-k} \xi\right)+\chi(\xi)$, and for $\lambda=2^{k}, k \geqslant 1$, we define $w_{\lambda}=Q_{k}(w)$, where $\hat{Q}_{k} w(\xi, \eta)=\eta\left(2^{-k} \xi\right) \hat{w}(\xi, \eta), w_{0}=Q_{0}(w)$, and $\widehat{Q_{0} w}(\xi, \eta)=\chi(\xi) \hat{w}(\xi, \eta)$. We first estimate $\left\|\partial_{x} w_{\lambda}\right\|_{L_{T}^{1} L_{x y}^{\infty}}$ : let us assume, for simplicity, that $T=1$. Split $[0,1]=\bigcup_{j} I_{j}$, where $I_{j}=\left[a_{j}, b_{j}\right]$ and $b_{j}-a_{j}=1 / \lambda, j=1, \ldots, \lambda$. Then,

$$
\left\|\partial_{x} w_{\lambda}\right\|_{L_{T}^{1} L_{x y}^{\infty}} \leqslant \sum_{j}\left\|\partial_{x} w_{\lambda}\right\|_{L_{I_{j}}^{1} L_{x y}^{\infty}} \leqslant \lambda \sum_{j}\left\|w_{\lambda}\right\|_{L_{I_{j}}^{1} L_{x y}^{\infty}}
$$

where we have used that $\xi \eta\left(2^{-k} \xi\right)$ has inverse Fourier transform whose $L^{1}$ norm in $x$ is bounded by $C \lambda$. We now use Cauchy-Schwartz in $I_{j}$, and continue with

$$
\lesssim \lambda^{1 / 2} \sum_{j}\left\|w_{\lambda}\right\|_{L_{I_{j}}^{2} L_{x y}^{\infty}}
$$

We now apply Duhamel's formula, in each $I_{j}$, to obtain, for $t \in I_{j}$,

$$
w_{\lambda}(t)=U\left(t-a_{j}\right) w_{\lambda}\left(-, a_{j}\right)+\int_{a_{j}}^{t} U\left(t-t^{\prime}\right) F_{\lambda}\left(t^{\prime}\right) d t^{\prime} .
$$

We use Corollary 1.6, and continue with

$$
\begin{aligned}
& \lesssim \lambda^{1 / 2} \sum_{j}\left(\left\|J_{x}^{\delta} w_{\lambda}\left(-, a_{j}\right)\right\|_{L^{2}}+\left\|D_{y}^{\delta} w_{\lambda}\left(-, a_{j}\right)\right\|_{L^{2}}\right)+\lambda^{1 / 2} \sum_{j} \int_{I_{j}}\left\|J_{x}^{\delta} F_{\lambda}\right\|_{L^{2}}+\left\|D_{y}^{\delta} F_{\lambda}\right\|_{L^{2}} \\
& \lesssim \sup _{t \in[0,1]}\left\|J_{x}^{3 / 2+\delta} w_{\lambda}(-, t)\right\|_{L^{2}}+\left\|J_{x}^{3 / 2} D_{y}^{\delta} w_{\lambda}(-, t)\right\|_{L^{2}}+\int_{0}^{t}\left\|J_{x}^{1 / 2+\delta} F_{\lambda}\right\|_{L^{2}}+\left\|J_{x}^{1 / 2} D_{y}^{\delta} F_{\lambda}\right\|_{L^{2}} .
\end{aligned}
$$

Write now $w_{1}=\sum_{k \geqslant 1} Q_{k}(w)$, so that $w=w_{1}+w_{0}$, and $w_{1}=\sum_{\lambda} w_{\lambda}$. Then, 


$$
\begin{aligned}
\left\|\partial_{x} w_{1}\right\| \leqslant & \sum_{\lambda}\left\|\partial_{x} w_{\lambda}\right\|_{L_{T}^{1} L_{x y}^{\infty}} \lesssim \sum_{k \geqslant 1} \sup \left(\left\|J_{x}^{3 / 2+\delta} Q_{k}(w)\right\|_{L^{2}}+\left\|J_{x}^{3 / 2} D_{y}^{\delta} Q_{k}(w)\right\|_{L^{2}}\right) \\
& +\int_{0}^{T}\left\|J_{x}^{\frac{1}{2}+\delta} Q_{k}(F)\right\|_{L^{2}}+\left\|J_{x}^{1 / 2} D_{y}^{\delta} Q_{k}(F)\right\|_{L^{2}} \\
\leqslant & \sum_{k \geqslant 1} 2^{-k \delta}\left\{\sup _{t}\left(\left\|J_{x}^{3 / 2+2 \delta} Q_{k}(w)\right\|_{L^{2}}+\left\|J_{x}^{3 / 2+\delta} D_{y}^{\delta} Q_{k}(w)\right\|_{L^{2}}\right)\right. \\
& \left.+\int_{0}^{T}\left\|J_{x}^{\frac{1}{2}+2 \delta} Q_{k}(F)\right\|_{L^{2}}+\left\|J_{x}^{1 / 2+\delta} D_{y}^{\delta} Q_{k}(F)\right\|_{L^{2}}\right\},
\end{aligned}
$$

and the desired estimate for $w_{1}$ follows. The proof of the estimate for $w_{0}$ is simpler, and Lemma 1.7 follows.

Lemma 1.8 (Leibniz rule for fractional differentiation).

(i) For $0<\alpha<1$,

$$
\left\|D_{x}^{\alpha}(f g)\right\|_{L^{2}(\mathbb{R})} \leqslant C\left\{\left\|D_{x}^{\alpha} f\right\|_{L^{p_{1}(\mathbb{R})}}\|g\|_{L^{q_{1}(\mathbb{R})}}+\|f\|_{L^{r_{1}(\mathbb{R})}}\left\|D_{x}^{\alpha} g\right\|_{L^{s_{1}(\mathbb{R})}}\right\}
$$

with $\frac{1}{2}=\frac{1}{p_{1}}+\frac{1}{q_{1}}=\frac{1}{r_{1}}+\frac{1}{s_{1}}, 1<p_{1}, q_{1}, r_{1}, s_{1} \leqslant \infty$. (See, for example, [11].)

(ii) For $0<\alpha, \beta<1$, we have

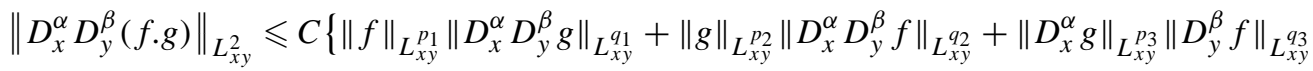

$$
\begin{aligned}
& \left.+\left\|D_{y}^{\beta} g\right\|_{L_{x y}^{p}}\left\|D_{x} \alpha f\right\|_{L_{x y}^{q_{4}}}\right\} \text {, }
\end{aligned}
$$

where $\frac{1}{2}=\frac{1}{p_{i}}+\frac{1}{q_{i}}, 1<p_{i} \leqslant \infty, 1<q_{i} \leqslant \infty$.

The proof of (ii) reduces, using an argument similar to the one given in [9] to a (now two parameter) version of the multilinear theorem of Coifman and Meyer [3]. The proof of (ii) is given in [16] (see also [8]).

\section{A priori estimates}

In this section we prove:

Lemma 2.1. Let $u$ be a solution to (1.2), with $u_{0} \in H_{-1}^{\infty}\left(\mathbb{R}^{2}\right) \cap Z$, defined, for all T. Let

$$
\left\|u_{0}\right\|_{Y_{s}}=\left\|J_{x}^{s} u_{0}\right\|_{L_{x y}^{2}}+\left\|\left(\partial_{x}^{-1} \partial_{y} u_{0}\right)\right\|_{L_{x y}^{2}} .
$$

Then, for any $s>3 / 2$, there exists $T=T_{s}$, depending only on $\left\|u_{0}\right\|_{Y_{s}}, s$, and a constant $C_{T}$, depending only on $\left\|u_{0}\right\|_{Y_{s}}$, s, so that

$$
\int_{0}^{T}\left\|\partial_{x} u\right\|_{L_{x y}^{\infty}} d t+\int_{0}^{T}\|u\|_{L_{x y}^{\infty}} d t \leqslant C_{T} .
$$


Proof. Let $s=\frac{3}{2}+\delta_{0}$, and choose $\delta>0$ so that $2 \delta \ll \delta_{0}$. Let us define

$$
f(T)=\left\|\partial_{x} u\right\|_{L_{T}^{1} L_{x y}^{\infty}}+\|u\|_{L_{T}^{1} L_{x y}^{\infty}}=\int_{0}^{T}\left(\left\|\partial_{x} u\right\|_{L_{x y}^{\infty}}+\|u\|_{L_{x y}^{\infty}}\right) d t .
$$

We will prove the estimate

$$
f(T) \leqslant C\left\|u_{0}\right\|_{Y_{s}}[\exp (C f(T))+1]
$$

for a fixed, universal constant $C$.

Let us take (2.4) temporarily for granted, and let us conclude the proof of (2.2). We first note that there exists $\varepsilon_{0}>0$ such that, if $\left\|u_{0}\right\|_{Y_{s}} \leqslant \varepsilon_{0}$, then (2.4) implies that $f(1) \leqslant M$, for a universal constant $M$. This follows from a standard continuity argument. (For instance, let $F(X, \varepsilon)=X-C \varepsilon \exp (C x)-C \varepsilon$. Note that $F(0,0)=0$, and that $\frac{\partial F}{\partial X}(0,0)=1, \frac{\partial F}{\partial \varepsilon}(0,0)=-2 C$. The implicit function theorem now guarantees that for $0<\varepsilon<\varepsilon_{0}$, there exists a smooth function $A(\varepsilon)$, which is increasing in $\varepsilon$, so that $F(A(\varepsilon), \varepsilon)=0$. If $M=A\left(\left\|u_{0}\right\|_{Y_{s}}\right)$, and $\left\|u_{0}\right\|_{Y_{s}} \leqslant \varepsilon_{0}$, it is easy to see that $f(1) \leqslant M$.) Next, note that $u$ is a solution of (1.2), with initial data $u_{0}$, if and only if $u_{\lambda}(x, y, t)=$ $\lambda^{2} u\left(\lambda x, \lambda^{2} y, \lambda^{3} t\right)$ is a solution of (1.2) with initial data $u_{0, \lambda}(x, y)=\lambda^{2} u_{0}\left(\lambda x, \lambda^{2} y\right)$. Since $\left\|u_{0, \lambda}\right\|_{L^{2}}=\lambda^{1 / 2}\left\|u_{0}\right\|_{L^{2}}$, $\left\|D_{x}^{s} u_{0, \lambda}\right\|_{L^{2}}=\lambda^{\frac{1}{2}+s}\left\|D_{x}^{s} u_{0}\right\|_{L^{2}}$ and $\left\|\partial_{x}^{-1} \partial_{y} u_{0, \lambda}\right\|_{L^{2}}=\lambda^{1+1 / 2}\left\|\partial_{x}^{-1} \partial_{y} u_{0}\right\|_{L^{2}}$, we can first choose $\lambda=\lambda\left(\left\|u_{0}\right\|_{Y^{s}}\right)$ so that $\left\|u_{0, \lambda}\right\|_{Y_{s}} \leqslant \varepsilon_{0}$, and apply the above conclusion to $u_{\lambda}$, to obtain (2.2).

We now turn to the proof of (2.4): we will use Lemma 1.7, and write (1.2) as

$$
\partial_{t} u+\partial_{x}^{3} u-\partial_{x}^{-1} \partial_{y}^{2} u=-u \partial_{x} u .
$$

We then obtain:

$$
\begin{aligned}
\left\|\partial_{x} u\right\|_{L_{T}^{1} L_{x y}^{\infty} \leqslant} \leqslant & C_{\delta, T}\left[\sup _{0<t<T}\left\|J_{x}^{3 / 2+2 \delta} u\right\|_{L^{2}}+\sup _{0<t<T}\left\|J_{x}^{3 / 2+\delta} D_{y}^{\delta} u\right\|_{L^{2}}\right] \\
& +C_{\delta, T} \int_{0}^{T}\left\|J_{x}^{1 / 2+2 \delta}\left(u \partial_{x} u\right)\right\|_{L^{2}}+C_{\delta, T} \int_{0}^{T}\left\|J_{x}^{\frac{1}{2}+\delta} D_{y}^{\delta}\left(u \partial_{x} u\right)\right\|_{L^{2}} \\
= & \mathrm{I}+\mathrm{II}+\mathrm{III}+\mathrm{IV} .
\end{aligned}
$$

To bound I, we use Lemma 1.3 to see that

$$
\mathrm{I} \leqslant C_{s} \exp \left(C_{s} \int_{0}^{T}\left\|\partial_{x} u\right\|_{L_{x y}^{\infty}}\right)\left\|u_{0}\right\|_{Y_{s}}
$$

To bound II, we use Plancherel's theorem, and the fact that $a b \leqslant \frac{a^{q}}{q}+\frac{b^{p}}{p}, \frac{1}{p}+\frac{1}{q}=1$, to see that

$$
(1+|\xi|)^{\frac{3}{2}+\delta} \cdot|\eta|^{\delta}=(1+|\xi|)^{\frac{3}{2}+\delta} \cdot|\xi|^{\delta} \cdot\left(\frac{|\eta|}{|\xi|}\right)^{\delta} \leqslant(1+|\xi|)^{\left(\frac{3}{2}+2 \delta\right)}\left(\frac{|\eta|}{|\xi|}\right)^{\delta} \lesssim(1+|\xi|)^{\left(\frac{3}{2}+2 \delta\right) /(1-\delta)}+\frac{|\eta|}{|\xi|}
$$

where we have chosen $q=1 / \delta, p=1 /(1-\delta)$. If now $\delta$ is chosen so small that $\left(\frac{3}{2}+2 \delta\right) /(1-\delta) \leqslant \frac{3}{2}+\delta_{0}$, we see that II $\lesssim \sup _{0<t<T}\|u\|_{Y_{s}}$, and in view of Lemma 1.3,

$$
\mathrm{II} \leqslant C_{s} \exp \left(C_{s} \int_{0}^{T}\left\|\partial_{x} u\right\|_{L_{x y}^{\infty}}\right)\left\|u_{0}\right\|_{Y_{s}} .
$$


In order to estimate III, we use that $\left\|J_{x}^{1 / 2+2 \delta}(f)\right\|_{L^{2}} \leqslant\|f\|_{L^{2}}+\left\|D_{x}^{1 / 2+2 \delta}(f)\right\|_{L^{2}}$, to bound III by $\mathrm{III}_{1}+\mathrm{III}_{2}$. For III $_{1}$ we see that it is bounded by $\sup _{0<t<T}\|u\|_{L^{2}} \cdot \int_{0}^{T}\left\|\partial_{x} u\right\|_{L^{\infty}}$, and hence it has the desired bound because Lemma 1.3 gives $\sup _{0<t<T}\|u\|_{L^{2}} \leqslant C\left\|u_{0}\right\|_{Y_{s}}$. $\exp C_{s} \int_{0}^{T}\left\|\partial_{x} u\right\|_{L^{\infty}}$.

For $\mathrm{III}_{2}$, we use Lemma 1.8(i), to obtain:

$$
\begin{aligned}
\mathrm{III}_{2} \lesssim & \int_{0}^{T}\left\|D_{x}^{\frac{1}{2}+2 \delta} \partial_{x} u\right\|_{L^{2}}\|u\|_{L^{\infty}}+\left\|\partial_{x} u\right\|_{L^{\infty}}\left\|D_{x}^{\frac{1}{2}+2 \delta} u\right\|_{L^{2}} \\
\lesssim & \text { (in view of Lemma 1.3) } C_{s}\left\|u_{0}\right\|_{Y_{s}} \exp \left(C_{s} \int_{0}^{T}\left\|\partial_{x} u\right\|_{L^{\infty}}\right) \\
& \times \int_{0}^{T}\|u\|_{L^{\infty}}+C_{s}\left\|u_{0}\right\|_{Y_{s}} \int_{0}^{T}\left\|\partial_{x} u\right\|_{L^{\infty}} \exp \left(C_{s} \int_{0}^{T}\left\|\partial_{x} u\right\|_{L^{\infty}}\right) \\
\leqslant & C_{S}\left\|u_{0}\right\|_{Y_{s}} \exp \left(C_{S} f(T)\right) .
\end{aligned}
$$

Finally, for IV, we bound it first by $\int_{0}^{T}\left\|D_{y}^{\delta}\left(u \partial_{x} u\right)\right\|_{L^{2}}+\int_{0}^{T}\left\|D_{x}^{\frac{1}{2}+\delta} D_{y}^{\delta}\left(u \partial_{x} u\right)\right\|_{L^{2}}=\mathrm{IV}_{1}+\mathrm{IV}_{2}$. To bound $\mathrm{IV}_{1}$, we use Lemma 1.8(i), in the $y$ variable, to see that

$$
\mathrm{IV}_{1} \lesssim \int_{0}^{T}\left\|D_{y}^{\delta}(u)\right\|_{L^{2}}\left\|\partial_{x} u\right\|_{L^{\infty}}+\|u\|_{L^{\infty}}\left\|D_{y}^{\delta} \partial_{x} u\right\|_{L^{2}}=\operatorname{IV}_{1,1}+\mathrm{IV}_{1,2}
$$

To bound $\mathrm{IV}_{1,1}$, we will use Plancherel's theorem for the first factor, and the inequalities

$$
|\eta|^{\delta}=|\xi|^{\delta}\left(\frac{|\eta|}{|\xi|}\right)^{\delta} \leqslant(1+|\xi|)^{\delta}\left(\frac{|\eta|}{|\xi|}\right)^{\delta} \lesssim(1+|\xi|)^{\delta / 1-\delta}+\frac{|\eta|}{|\xi|}
$$

where $\delta$ is chosen so that $\delta /(1-\delta) \leqslant 3 / 2+\delta_{0}$. This shows that (using Lemma 1.3)

$$
\mathrm{IV}_{1,1} \leqslant C_{S}\left\|u_{0}\right\|_{Y_{S}} \exp C_{S} \int_{0}^{T}\left\|\partial_{x} u\right\|_{L^{\infty}} .
$$

For $\mathrm{IV}_{1,2}$ we proceed similarly, using that

$$
|\eta|^{\delta}|\xi|=|\xi|^{1+\delta}\left(\frac{|\eta|}{|\xi|}\right)^{\delta} \leqslant(1+|\xi|)^{1+\delta}\left(\frac{|\eta|}{|\xi|}\right)^{\delta} \leqslant(1+|\xi|)^{(1+\delta) /(1-\delta)}+\frac{|\eta|}{|\xi|},
$$

and choosing $\delta$ so that $(1+\delta) /(1-\delta) \leqslant 3 / 2+\delta_{0}$. This gives the bound $\mathrm{IV}_{1,2} \leqslant C_{s}\left\|u_{0}\right\|_{Y_{s}} \exp \left(C_{S} f(T)\right)$. We next turn to $\mathrm{IV}_{2}$, and use Lemma 1.8(ii) to bound it by

$$
\begin{aligned}
& \int_{0}^{T}\left\|\partial_{x} u\right\|_{L^{\infty}}\left\|D_{x}^{\frac{1}{2}+\delta} D_{y}^{\delta} u\right\|_{L^{2}}+\int_{0}^{T}\left\|\partial_{x} D_{x}^{\frac{1}{2}+\delta} D_{y}^{\delta} u\right\|_{L^{2}}\|u\|_{L^{\infty}} \\
& \quad+\int_{0}^{T}\left\|D_{x}^{\frac{1}{2}+\delta} u\right\|_{L^{\infty}}\left\|D_{y}^{\delta} \partial_{x} u\right\|_{L^{2}}+\int_{0}^{T}\left\|D_{y}^{\delta} u\right\|_{L^{p_{1}}\left\|D_{x}^{1 / 2+\delta} \partial_{x} u\right\|_{L^{q_{1}}},}
\end{aligned}
$$


where $\frac{1}{p_{1}}+\frac{1}{q_{1}}=\frac{1}{2}$ are to be determined. We call each one of these four terms $\operatorname{IV}_{2, i}$. To bound $\operatorname{IV}_{2,1}$, we again use Plancherel, and

$$
|\xi|^{\frac{1}{2}+\delta}|\eta|^{\delta} \leqslant(1+|\xi|)^{\frac{1}{2}+2 \delta}\left(\frac{|\eta|}{|\xi|}\right)^{\delta} \lesssim(1+|\xi|)^{\left(\frac{1}{2}+2 \delta\right) /(1-\delta)}+\frac{|\eta|}{|\xi|}
$$

and if $\left(\frac{1}{2}+2 \delta\right) /(1-\delta) \leqslant \frac{3}{2}+\delta_{0}$, Lemma 1.3 shows that

$$
\mathrm{IV}_{2,1} \leqslant C_{s}\left\|u_{0}\right\|_{Y_{s}} \exp \left(C_{s} \int_{0}^{T}\left\|\partial_{x} u\right\|_{L^{\infty}}\right) .
$$

For $\mathrm{IV}_{2,2}$ we proceed similarly, using that

$$
|\xi||\xi|^{\frac{1}{2}+2 \delta}\left(\frac{|\eta|}{|\xi|}\right)^{\delta} \lesssim(1+|\xi|)^{\left(\frac{3}{2}+2 \delta\right) /(1-\delta)}+\frac{|\eta|}{|\xi|},
$$

and the fact that $\left(\frac{3}{2}+2 \delta\right) /(1-\delta) \leqslant \frac{3}{2}+\delta_{0}$. We obtain, using Lemma 1.3 , that

$$
\mathrm{IV}_{2,2} \leqslant C_{s}\left\|u_{0}\right\|_{Y_{s}} \exp \left(C_{s} f(T)\right) .
$$

In order to bound $\mathrm{IV}_{2,3}$, we will use the following inequality $\left(\delta<\frac{1}{2}\right)$

$$
\left\|D_{x}^{\frac{1}{2}+\delta} u\right\|_{L_{x y}^{\infty}} \lesssim\|u\|_{L_{x y}^{\infty}}+\left\|\partial_{x} u\right\|_{L_{x y}^{\infty}} .
$$

To establish (2.5), let us use a Littlewood-Paley decomposition, as in the proof of Lemma 1.7, and write $u=$ $u_{0}+u_{1}$, as in that proof. In order to estimate $D_{x}^{\frac{1}{2}+\delta} u_{0}$, note that, if $\hat{f}(\xi)=|\xi|^{1 / 2+\delta} \chi(\xi)$, then $f \in L^{1}(\mathbb{R})$. Hence, $\left\|D_{x}^{\frac{1}{2}+\delta} u_{0}\right\|_{L_{x y}^{\infty}} \leqslant\|u\|_{L_{x y}^{\infty}}$. Moreover, in order to estimate $D_{x}^{\frac{1}{2}+\delta} u_{1}=\sum_{k \geqslant 1} D_{x}^{\frac{1}{2}+\delta} Q_{k} u$, note that

$$
\begin{aligned}
D_{x}^{\frac{1}{2}+\delta} \widehat{Q_{k}} u(\xi, \eta) & =|\xi|^{\frac{1}{2}+\delta} \eta\left(2^{-k} \xi\right) \hat{u}(\xi, \eta) \\
& =\frac{|\xi|^{\frac{1}{2}+\delta}}{\xi} \xi \eta\left(2^{-k} \xi\right) \hat{u}(\xi, \eta)=2^{-k\left(\frac{1}{2}-\delta\right)} \frac{\left|2^{-k} \xi\right|^{\frac{1}{2}+\delta}}{\left(2^{-k} \xi\right)} \eta\left(2^{-k} \xi\right) \xi \hat{u}(\xi, \eta),
\end{aligned}
$$

and that if $\hat{g}(\xi)=\left(|\xi|^{\frac{1}{2}+\delta} / \xi\right) \eta(\xi)$, then $g \in L^{1}(\mathbb{R})$. Thus, $\left\|D_{x}^{\frac{1}{2}+\delta} Q_{k} u\right\|_{L_{x y}^{\infty}} \leqslant 2^{-k\left(\frac{1}{2}-\delta\right)}\left\|\partial_{x} u\right\|_{L_{x y}^{\infty}}$, and (2.5) follows.

Because of (2.5), $\mathrm{IV}_{2,3}$ is bounded by

$$
\sup _{0<t<T}\left\|D_{y}^{\delta} \partial_{x} u\right\|_{L^{2}}\left\{\int_{0}^{T}\|u\|_{L^{\infty}}+\left\|\partial_{x} u\right\|_{L^{\infty}}\right\} .
$$

Using Plancherel, and

$$
|\eta|^{\delta}|\xi| \leqslant\left(\frac{|\eta|}{|\xi|}\right)^{\delta}(1+|\xi|)^{1+\delta} \lesssim \frac{|\eta|}{|\xi|}+(1+|\xi|)^{1+\delta /(1-\delta)}
$$

choosing $\delta$ so that $(1+\delta) /(1-\delta) \leqslant \frac{3}{2}+\delta_{0}$, and using Lemma 1.3, we see that

$$
\mathrm{IV}_{2,3} \leqslant C_{s}\left\|u_{0}\right\|_{Y_{s}} \exp \left(C_{s} f(T)\right) .
$$

To bound $\mathrm{IV}_{2,4}$, we need to prove first a couple of auxiliary estimates:

$$
\begin{aligned}
& \left\|D_{x}^{1 / 2+\delta} \partial_{x} u\right\|_{L_{T}^{s_{1}} L_{x y}^{q_{1}}} \lesssim\left\|\partial_{x} u\right\|_{L_{T}^{1} L_{x y}^{\infty}}^{\theta} \cdot\left\|J_{x}^{3 / 2+\delta_{0}} u\right\|_{L_{T}^{\infty} L_{x y}^{2}}^{1-\theta} \\
& \left\|D_{y}^{\delta} u\right\|_{L_{T}^{r_{1}} L_{x y}^{p_{1}}} \lesssim\left(\|u\|_{L_{T}^{1} L_{x y}^{\infty}}\right)^{\eta}\left(\left\|D_{y}^{1 / 2} u\right\|_{L_{T}^{\infty} L_{x y}^{2}}+\|u\|_{L_{T}^{\infty} L_{x y}^{2}}\right)^{1-\eta},
\end{aligned}
$$


where $q_{1}>2, s_{1}<\infty, \frac{1}{p_{1}}=\frac{1}{2}-\frac{1}{q_{1}}, \frac{1}{r_{1}}=1-\frac{1}{s_{1}}$ are suitably chosen, and $\theta, \eta$ are also suitably chosen. In order to establish (2.6), we use a Littlewood-Paley decomposition in $x$, as in the proof of Lemma 1.7. We then have $\partial_{x} u=$ $\sum_{k \geqslant 0} Q_{k}\left(\partial_{x} u\right)$, and $D_{x}^{\frac{1}{2}+\delta} \partial_{x} u=\sum_{k \geqslant 0} D_{x}^{\frac{1}{2}+\delta} Q_{k}\left(\partial_{x} u\right)$. Now, for $k \geqslant 1, D_{x}^{\frac{1}{2}+\delta} Q_{k}=2^{k\left(\frac{1}{2}+\delta\right)} \tilde{Q}_{k}$, where $\widehat{\tilde{Q}_{k}} f(\xi)=$ $\tilde{\eta}\left(2^{-k} \xi\right) \hat{f}(\xi)$, and $\tilde{\eta}$ has similar properties to $\eta$, and $D_{x}^{\frac{1}{2}+\delta} Q_{0}=\tilde{Q}_{0}$, where $\widehat{\tilde{Q}_{0}} f(\xi)=\tilde{\chi}(\xi) \hat{f}(\xi)$, and if $\hat{g}=\tilde{\chi}$, $g \in L^{1}(\mathbb{R})$. We then have:

$$
\left\|D_{x}^{1 / 2+\delta} \partial_{x} u\right\|_{L_{T}^{s_{1}} L_{x y}^{q_{1}}} \leqslant \sum_{k \geqslant 0} 2^{k\left(\frac{1}{2}+\delta\right)}\left\|\tilde{Q}_{k}\left(\partial_{x} u\right)\right\|_{L_{T}^{s_{1}} L_{x y}^{q_{1}}}
$$

We first bound the term $k=0$ in the right-hand side. Let $\frac{1}{s_{1}}=\theta, \frac{1}{q_{1}}=\frac{(1-\theta)}{2}$, where $0 \leqslant \theta \leqslant 1$. Then,

$$
\left\|\tilde{Q}_{0} \partial_{x} u\right\|_{L_{T}^{s_{1}} L_{x y}^{q_{1}}} \leqslant\left\|\tilde{Q}_{0} \partial_{x} u\right\|_{L_{T}^{1} L_{x y}^{\infty}}^{\theta} \cdot\left\|\tilde{Q}_{0} \partial_{x} u\right\|_{L_{T}^{\infty} L_{x y}^{2}}^{1-\theta},
$$

and this term has the correct control.

For $k \geqslant 1$, we again use the same choices of $s_{1}, q_{1}$, and use

$$
\begin{aligned}
\left\|\tilde{Q}_{k} \partial_{x} u\right\|_{L_{T}^{s_{1}} L_{x y}^{q_{1}}} & \leqslant\left\|\tilde{Q}_{k} \partial_{x} u\right\|_{L_{T}^{1} L_{x y}^{\infty}}^{\theta} \cdot\left\|\tilde{Q}_{k} \partial_{x} u\right\|_{L_{T}^{\infty} L_{x y}^{2}}^{1-\theta} \\
& \lesssim\left\|\tilde{Q}_{k} \partial_{x} u\right\|_{L_{T}^{1} L_{x y}^{\infty}}^{\theta} \cdot 2^{-k\left(\frac{1}{2}+\delta_{0}\right)(1-\theta)}\left\|\tilde{\tilde{Q}}_{k} D_{x}^{\left(\frac{1}{2}+\delta_{0}\right)} \partial_{x} u\right\|_{L_{t}^{\infty} L_{x y}^{2}}^{1-\theta} \\
& \lesssim\left\|\partial_{x} u\right\|_{L_{T}^{1} L_{x y}^{\infty}}^{\theta} \cdot 2^{-k\left(\frac{1}{2}+\delta_{0}\right)(1-\theta)}\left\|D_{x}^{\left(\frac{1}{2}+\delta_{0}\right)} \partial_{x} u\right\|_{L_{T}^{\infty} L_{x y}^{2}}^{1-\theta},
\end{aligned}
$$

where $\tilde{\tilde{Q}}_{k}$ is associated to $\tilde{\tilde{\eta}}\left(2^{-k} \xi\right)$, where $\tilde{\tilde{\eta}}$ has similar properties to $\tilde{\eta}$. From this, we obtain (2.6), as long as $\left(\frac{1}{2}+\right.$ $\delta)<\left(\frac{1}{2}+\delta_{0}\right)(1-\theta)$, or, choosing $\delta$ small enough, as long as $\frac{1}{2}<\left(\frac{1}{2}+\delta_{0}\right)(1-\theta)$, or $0<\theta<1-\left[\frac{1}{2} /\left(\frac{1}{2}+\delta_{0}\right)\right]=\eta_{0}$, and $\frac{1}{s_{1}}=\theta, \frac{1}{q_{1}}=(1-\theta) / 2$. We next turn to (2.7). This time we perform a similar Littlewood-Paley decomposition, but in the $y$ variable. We need $\frac{1}{p_{1}}=\frac{\theta}{2}, \frac{1}{r_{1}}=1-\theta$. We proceed in a similar manner, and are led to controlling

$$
\sum_{k \geqslant 0} 2^{\delta k}\left\|\tilde{Q}_{k} u\right\|_{L_{T}^{r_{1}} L_{x y}^{p_{1}}}
$$

where $\tilde{Q}_{k}$ acts on the $y$ variable. But, for $0 \leqslant \eta \leqslant 1, \frac{1}{r_{1}}=\eta, \frac{1}{p_{1}}=\frac{(1-\eta)}{2}$, we have

$$
\left\|\tilde{Q}_{k} u\right\|_{L_{t}^{r_{1}} L_{x y}^{p_{1}}} \leqslant\left\|\tilde{Q}_{k} u\right\|_{L_{t}^{1} L_{x y}^{\infty}}^{\eta}\left\|\tilde{Q}_{k} u\right\|_{L_{T}^{\infty} L_{x y}^{2}}^{1-\eta},
$$

and we see that, for (2.7) to hold, we need $\delta<\frac{1}{2}(1-\eta)$. Since $\eta=(1-\theta)$, we need $\delta<\frac{1}{2} \theta$, and $0<\theta<$ $1-\left[\frac{1}{2} /\left(\frac{1}{2}+\delta_{0}\right)\right]=\eta_{0}$, which we can clearly achieve.

Now, in order to bound $\mathrm{IV}_{2,4}$, we choose $p_{1}$ as in (2.7), $q_{1}$ as in (2.6). Observe also that, since

$$
|\eta|^{1 / 2} \leqslant\left(\frac{|\eta|^{1 / 2}}{|\xi|}\right)(1+|\xi|)^{1 / 2} \leqslant \frac{|\eta|}{|\xi|}+(1+|\xi|) \leqslant \frac{|\eta|}{|\xi|}+(1+|\xi|)^{\frac{3}{2}+\delta_{0}}
$$

we have $\left\|D_{y}^{1 / 2} u\right\|_{L_{T}^{\infty} L_{x y}^{2}}+\|u\|_{L_{T}^{\infty} L_{x y}^{2}} \leqslant \sup _{0<t<T}\|u\|_{Y_{s}}$. We then have (recalling that $\eta=1-\theta$ ):



$$
\begin{aligned}
& \lesssim C_{s}\left\|u_{0}\right\|_{Y_{s}}\left\|\partial_{x} u\right\|_{L_{T}^{1} L_{x y}^{\infty}}^{\theta}\|u\|_{L_{T}^{1} L_{x y}^{\infty}}^{1-\theta} \exp \left(C_{s}\left\|\partial_{x} u\right\|_{L_{t}^{1} L_{x y}^{\infty}}\right) \lesssim C_{s}\left\|u_{0}\right\|_{Y_{S}} \exp \left(C_{s} f(T)\right),
\end{aligned}
$$

where in the next to last inequality we have used Lemma 1.3. 
All in all, we have shown that

$$
\left\|\partial_{x} u\right\|_{L_{T}^{1} L_{x y}^{\infty}} \leqslant C_{s}\left\|u_{0}\right\|_{Y_{s}} \exp \left(C_{S} f(T)\right) .
$$

We now turn to the bound for $\|u\|_{L_{T}^{1} L_{x y}^{\infty}}$. We use Duhamel's formula, and write

$$
u(t)=U(t) u_{0}+\int_{0}^{t} U\left(t-t^{\prime}\right) u \partial_{x} u d t^{\prime} .
$$

We apply Corollary 1.6, and Minkowski's integral inequality, to see that

$$
\|u\|_{L_{T}^{1} L_{x y}^{\infty}} \leqslant C_{T}\left(\left\|u_{0}\right\|_{Y_{s}}+\int_{0}^{T}\left[\left\|u \partial_{x} u\right\|_{L^{2}}+\left\|D_{x}^{\varepsilon}\left(u \partial_{x} u\right)\right\|_{L^{2}}+\left\|D_{y}^{\varepsilon}\left(u \partial_{x} u\right)\right\|_{L^{2}}\right]\right) .
$$

We proceed to estimate the three terms in the integral, which we call $A+B+C$.

$$
\begin{aligned}
A \leqslant\|u\|_{L_{T}^{1} L_{x y}^{\infty}} \sup _{0<t<T}\left\|\partial_{x} u\right\|_{L_{x y}^{2}} & \leqslant C_{s}\left\|u_{0}\right\|_{Y_{s}} \exp \left(C_{s}\left\|\partial_{x} u\right\|_{L_{x y}^{\infty}}\right) \cdot\|u\|_{L_{T}^{1} L_{x y}^{\infty}} \\
& \leqslant C_{s}\left\|u_{0}\right\|_{Y_{s}} \exp \left(C_{s} f(T)\right),
\end{aligned}
$$

where in the next to last inequality we used Lemma 1.3. For $B$, we use first Lemma 1.8(i) and obtain:

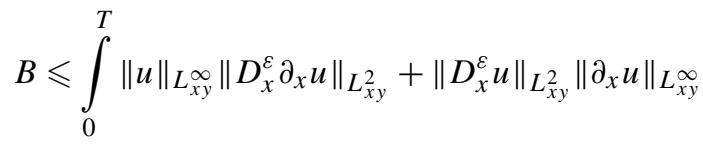

$$
\begin{aligned}
& \lesssim \sup _{0<t<T}\|u\|_{Y_{s}} \cdot\left(\|u\|_{L_{T}^{1} L_{x y}^{\infty}}+\left\|\partial_{x} u\right\|_{L_{T}^{1} L_{x y}^{\infty}}\right) \\
& \leqslant C_{s}\left\|u_{0}\right\|_{Y_{s}} \exp \left(C_{s}\left\|\partial_{x} u\right\|_{L_{T}^{1} L_{x y}^{\infty}}\right)\left(\|u\|_{L_{T}^{1} L_{x y}^{\infty}}+\left\|\partial_{x} u\right\|_{L_{T}^{1} L_{x y}^{\infty}}\right) \\
& \leqslant C_{S}\left\|u_{0}\right\|_{Y_{S}} \exp \left(C_{S} f(T)\right) \text {, }
\end{aligned}
$$

where we have used Lemma 1.3 to bound $\sup _{0<t<T}\|u\|_{Y_{s}}$.

For $C$, we use Lemma 1.8(i) in the $y$-variable, and obtain:

$$
C \lesssim \int_{0}^{T}\|u\|_{L_{x y}^{\infty}}\left\|D_{y}^{\varepsilon} \partial_{x} u\right\|_{L_{x y}^{2}}+\left\|D_{y}^{\varepsilon} u\right\|_{L_{x y}^{2}}\left\|\partial_{x} u\right\|_{L_{x y}^{\infty}}
$$

and since

$$
|\eta|^{\varepsilon}|\xi|=\left(\frac{|\eta|}{|\xi|}\right)^{\varepsilon}\left(|\xi|^{1+\varepsilon}\right) \leqslant\left(\frac{|\eta|}{|\xi|}\right)^{\varepsilon}(1+|\xi|)^{1+\varepsilon} \leqslant \frac{|\eta|}{|\xi|}+(1+|\xi|)^{(1+\varepsilon) /(1-\varepsilon)}
$$

and we can choose $\varepsilon$ so that $(1+\varepsilon) /(1-\varepsilon) \leqslant \frac{3}{2}+\delta_{0}$, a similar argument gives $C \lesssim C_{s}\left\|u_{0}\right\|_{Y_{s}} \exp \left(C_{s} f(T)\right)$, and so

$$
\|u\|_{L_{T}^{1} L_{x y}^{\infty}} \lesssim C_{T}\left\|u_{0}\right\|_{Y_{s}}+C_{s}\left\|u_{0}\right\|_{Y_{s}} \cdot \exp \left(C_{S} f(T)\right),
$$

which, together with (2.9) gives (2.4), and hence Lemma 2.1. 


\section{Local and global well-posedness}

Our first result, a local well-posedness one in the space $Y_{s}, s>3 / 2$, follows readily from Lemmas 1.3 and 2.1.

Theorem 3.1. The (IVP) (2.1) is locally well-posed in $Y_{s}, s>3 / 2$. More precisely, given $u_{0} \in Y_{s}, s>3 / 2$, there exists $T=T\left(\left\|u_{0}\right\|_{Y_{s}}\right)$, and a unique solution $u$ to (1.2), such that $u \in C\left([0, T]: Y_{s}\right), u, \partial_{x} u \in L_{T}^{1} L_{x y}^{\infty}$. Moreover, the mapping $u_{0} \mapsto u \in C\left([0, T] ; Y_{S}\right)$ is continuous.

Proof. Let $u_{0} \in Y_{s}$, find $u_{0, \varepsilon} \in Y_{S} \cap H_{-1}^{\infty}\left(\mathbb{R}^{2}\right) \cap Z$, such that $\left\|u_{0}-u_{0, \varepsilon}\right\|_{Y_{s}} \rightarrow 0$, and $\left\|u_{0, \varepsilon}\right\|_{Y_{s}} \leqslant 2\left\|u_{0}\right\|_{Y_{s}}$. Let now $u_{\varepsilon}$ be the solutions associated to $u_{0, \varepsilon}$, guaranteed by Lemma 1.1. Note that Lemma 2.1 gives us $T=T\left(\left\|u_{0}\right\|_{Y_{s}}\right)$ so that

$$
\left\|\partial_{x} u_{\varepsilon}\right\|_{L_{T}^{1} L_{x y}^{\infty}}+\left\|u_{\varepsilon}\right\|_{L_{T}^{1} L_{x y}^{\infty}} \leqslant C_{T} .
$$

Lemma 1.3 now guarantees that

$$
\sup _{0<t<T}\left\|u_{\varepsilon}\right\|_{Y_{s}} \leqslant C_{T} .
$$

Next, a use of Gronwall's inequality, combined with (3.2), shows that, as $\varepsilon, \varepsilon^{\prime} \rightarrow 0 \sup _{0<t<T}\left\|u_{\varepsilon}-u_{\varepsilon^{\prime}}\right\|_{L^{2}} \rightarrow 0$. hence, we can find $u \in C\left([0, T] ; Y_{s^{\prime}}\right) \cap L^{\infty}\left([0, T] ; Y_{s}\right),\left(s^{\prime}<s\right)$ such that $u_{\varepsilon} \rightarrow u$ in $C\left([0, T], Y_{s^{\prime}}\right)$. The fact that $u$ is a solution to (1.2) is now clear. The uniqueness of $u$ follows from the same use of Gronwall's inequality as above. Finally, the continuity of $u(t)$ in $Y_{s}$, and the continuity of the flow map in $Y_{s}$ are consequences of the well-known Bona and Smith [1] argument.

We now introduce the space $Z_{0}$, related to the conservation laws associated with KP-I.

$$
\begin{aligned}
& Z_{0}=\left\{\phi \in L^{2}\left(\mathbb{R}^{2}\right):\|\phi\|_{Z_{0}}<+\infty\right\}, \quad \text { where } \\
& \|\phi\|_{Z_{0}}=\|\phi\|_{L^{2}}+\left\|\partial_{x}^{2} \phi\right\|_{L^{2}}+\left\|\partial_{x} \phi\right\|_{L^{2}}+\left\|\partial_{x}^{-1} \partial_{y} \phi\right\|_{L^{2}}+\left\|\partial_{x}^{-2} \partial_{y}^{2} \phi\right\|_{L^{2}} .
\end{aligned}
$$

Note that, since $\partial_{y} \phi=\partial_{x} \partial_{x}^{-1} \partial_{y} \phi$, we have that $\left\|\partial_{y} \phi\right\|_{L^{2}} \lesssim\|\phi\|_{Z_{0}}$.

Theorem 3.4. Let $u_{0} \in Z_{0}$. then there exists a unique global solution $u$ of (1.2) with initial data $u_{0}$, such that $u \in L^{\infty}\left(\mathbb{R}_{+} ; Z_{0}\right)$.

Proof. Find $u_{0, \varepsilon}$ as in the proof of Theorem 3.1, which converge to $u_{0}$ in $Z_{0}$. Recall from [14] the conserved quantities

$$
\begin{aligned}
M(\phi)= & \int|\phi|^{2}, \\
E(\phi)= & \frac{1}{2} \int\left(\partial_{x} \phi\right)^{2}+\frac{1}{2} \int\left(\partial_{x}^{-1} \partial_{y} \phi\right)^{2}-\frac{1}{6} \int u^{3}, \\
F(\phi)= & \frac{3}{2} \int\left(\partial_{x}^{2} \phi\right)^{2}+5 \int\left(\partial_{y} \phi\right)^{2}+\frac{5}{6} \int\left(\partial_{x}^{-2} \partial_{y}^{2} \phi\right)^{2}-\frac{5}{6} \int \phi^{2} \partial_{x}^{-2} \partial_{y}^{2} \phi \\
& -\frac{5}{6} \int \phi\left(\partial_{x}^{-1} \partial_{y} \phi\right)^{2}+\frac{5}{4} \int \phi^{2} \partial_{x x} \phi+\frac{5}{24} \int \phi^{4} .
\end{aligned}
$$

Let $u_{\varepsilon}$ be the global solutions to (1.2) given by Lemma 1.1. Recall from Proposition 4 of [14] that $M\left(u_{\varepsilon}(t)\right)=$ $M\left(u_{0, \varepsilon}\right), E\left(u_{\varepsilon}(t)\right)=E\left(u_{0, \varepsilon}\right), F\left(u_{\varepsilon}(t)\right)=F\left(u_{0, \varepsilon}\right)$. As in [14], Proposition 5, we conclude that

$$
\left\|\partial_{x} u_{\varepsilon}(t)\right\|_{L^{2}}+\left\|\partial_{x}^{-1} \partial_{y} u_{\varepsilon}(t)\right\|_{L^{2}} \lesssim E\left(u_{0, \varepsilon}\right)+\left\|u_{0, \varepsilon}\right\|_{L^{2}}^{6}
$$


and $E\left(u_{0, \varepsilon}\right) \lesssim C\left(\left\|u_{0}\right\|_{Z_{0}}\right)$, and that $\left\|u_{\varepsilon}(t)\right\|_{L^{q}\left(\mathbb{R}^{2}\right)} \leqslant C\left(\left\|u_{0, \varepsilon}\right\|_{L^{2}}, E\left(u_{0, \varepsilon}\right)\right), q \in[2,6]$. Moreover, we also see that

$$
\left\|\partial_{x}^{2} u_{\varepsilon}(t)\right\|_{L^{2}}^{2}+\left\|\partial_{x}^{-2} \partial_{y}^{2} u_{\varepsilon}(t)\right\|_{L^{2}}^{2}+\left\|\partial_{y} u_{\varepsilon}(t)\right\|_{L^{2}}^{2} \leqslant C\left(\left\|u_{0}\right\|_{Z_{0}}\right)
$$

This shows that

$$
\left\|u_{\varepsilon}(t)\right\|_{Z_{0}} \leqslant C\left(\left\|u_{0}\right\|_{Z_{0}}\right) \quad \text { for all } t
$$

Note also that, for $\frac{3}{2}<s \leqslant 2$, we have $\|\phi\|_{Y_{s}} \leqslant\|\phi\|_{Z_{0}}$. As in the proof of Theorem 3.1, we can now construct a unique global solution $u \in C\left(\mathbb{R}^{+} ; Y_{s}\right), \frac{3}{2}<s \leqslant 2$, with $u \in L^{\infty}\left(\mathbb{R}^{+} ; Z_{0}\right)$.

Remark 3.5. One can give an improvement of Theorem 3.1 in the spirit of [10], by also establishing a 'local smoothing' estimate of Kato-type for KP-I. The resulting result would be the analog of Theorem 3.1, for $s>5 / 4$, but, since it would be quite intricate technically, and still falls short of the desired local well-posedness for $Y_{1}$, we have decided not to include this improvement.

\section{Acknowledgements}

The author is very grateful to N. Tzvetkov for his encouragement, and for pointing out that the global wellposedness result given here is a consequence of our local well-posedness result and [14]. The author is also grateful to J. Colliander, G. Staffilani and S. Ziesler for many conversations on KP-I equations, and to J. Pipher for useful discussions on Lemma 1.8(ii).

\section{References}

[1] J.L. Bona, R. Smith, The initial value problem for the Korteweg-de Vries equation, Philos. Trans. Roy. Soc. London Ser. A 278 (1975) 555-601.

[2] J. Bourgain, On the Cauchy problem for the Kadomstev-Petviashvili equation, Geom. Funct. Anal. 3 (1993) 315-341.

[3] R. Coifman, Y. Meyer, Au delà des operateurs pseudodifferéntiels, Astérisque 57 (1978).

[4] J. Colliander, C. Kenig, G. Staffilani, Small solutions for the Kadomstev-Petviashvili I equation, Mosc. Math. J. 1 (4) (2001) $491-520$.

[5] J. Colliander, C. Kenig, G. Staffilani, Low regularity solutions for the Kadomstev-Petviashvili I equation, Geom. Funct. Anal., submitted for publication.

[6] J. Colliander, C. Kenig, G. Staffilani, Corrections to: On solutions for the Kadomstev-Petviashvili I equation, Mosc. Math. J., submitted for publication.

[7] R.J. Iorio Jr., W.V.L. Nunes, On equations of KP-type, Proc. Roy. Soc. Edinburgh Sect. A 128 (1998) 725-743.

[8] J.L. Journé, Two problems of Calderón-Zygmund theory on product-spaces, Ann. Inst. Fourier Grenoble 38 (1) (1988) $111-132$.

[9] T. Kato, G. Ponce, Commutator estimates and the Euler and Navier-Stokes equations, Comm. Pure Appl. Math. 41 (1988) $891-907$.

[10] C. Kenig, K. Koenig, On the local well-posedness of the Benjamin-Ono and modified Benjamin-Ono equations, MRL, submitted for publication.

[11] C. Kenig, G. Ponce, L. Vega, Well-posedness and scattering results for the generalized Korteweg-de Vries equation via the contraction principle, Comm. Pure Appl. Math. 46 (1993) 527-620.

[12] H. Koch, N. Tzvetkov, Local well-posedness of the Benjamin-Ono equation in $H^{s}(\mathbb{R})$, IMRN 26 (2003) 1449-1464.

[13] L. Molinet, J.-C. Saut, N. Tzvetkov, Well-posedness and ill-posedness results for the Kadomstev-Petviashvili I equation, Duke Math. J. 115 (2) (2002) 353-384.

[14] L. Molinet, J.-C. Saut, N. Tzvetkov, Global well-posedness for the KP-I equation, Math. Annalen 324 (2002) 255-275.

[15] L. Molinet, J.-C. Saut, N. Tzvetkov, Correction: Global well-posedness for the KP-I equation, Math. Annalen, submitted for publication.

[16] C. Muscalu, J. Pipher, T. Tao, C. Thiele, Bi-parameter paraproducts, preprint.

[17] J.-C. Saut, Remarks on the generalized Kadomstev-Petviashvili equations, Indiana Univ. Math. J. 42 (1993) 1011-1026.

[18] H. Takaoka, Time local well-posedness for the Kadomstev-Petviashvili II equation, Harmonic Anal. Nonlin. PDE 1102 (1999) 1-8.

[19] N. Tzvetkov, Global low regularity solutions for Kadomstev-Petviashvili equation, Differential Integral Equations 13 (2000) 1289-1320.

[20] H. Takaoka, N. Tzvetkov, On the local regularity of Kadomstev-Petviashvili-II equation, IMRN 8 (2001) 77-114. 